\title{
Umbilical vein variation - case of an umbilical vein draining into the portal venous system
}

\author{
A Ahmed, MB BCh, FCRad(D) \\ Division of Radiology, Department of Radiation Sciences, Chris Hani \\ Baragwanath Hospital, Johannesburg \\ G Pitcher, MB BCh, FCS(SA) \\ Department of Paediatric Surgery, Chris Hani Baragwanath Hospital, \\ Johannesburg
}

Umbilical venous anomalies are not a common occurrence, and when encountered, they encompass a spectrum of variations. In the case presented, the possibility of an umbilical venous anomaly was raised on the abdominal X-ray done soon after birth. The neonate had a venous catheter inserted for vascular access. On the film done thereafter, the venous catheter was seen to deviate to the left of the midline (Fig. 1). The child subsequently underwent a laparotomy. No falciform ligament was demonstrated, the ligamentum venosum was absent and the umbilical vein was seen to drain directly into the superior mesenteric vein (Fig. 2).

The umbilical veins are a pair of developmental vessels that carry oxygenated blood between the placenta and the heart. From the fifth week of gestation, part of the umbilical veins becomes incorporated into

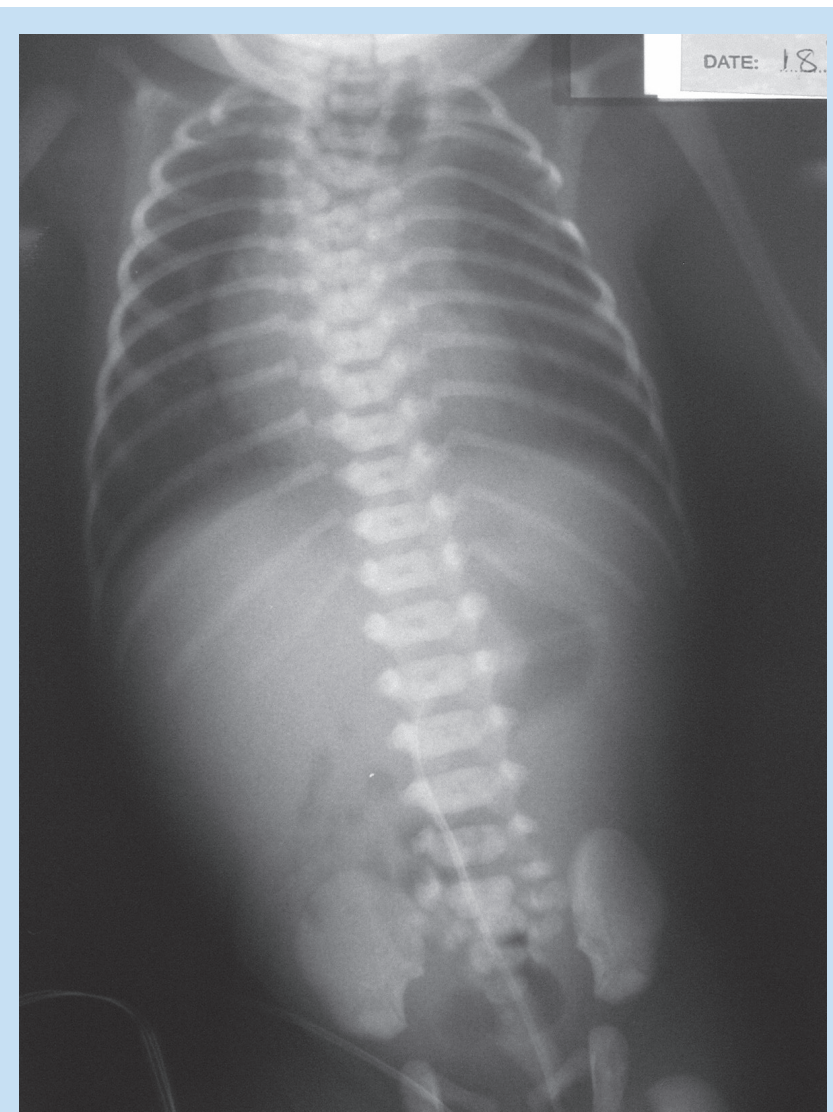

Fig. 1. Abdominal and chest $X$-ray demonstrating deviation of the umbilical venous line, curled up nasogastric tube in the oesophageal pouch and dorsal spine hemivertebrae.

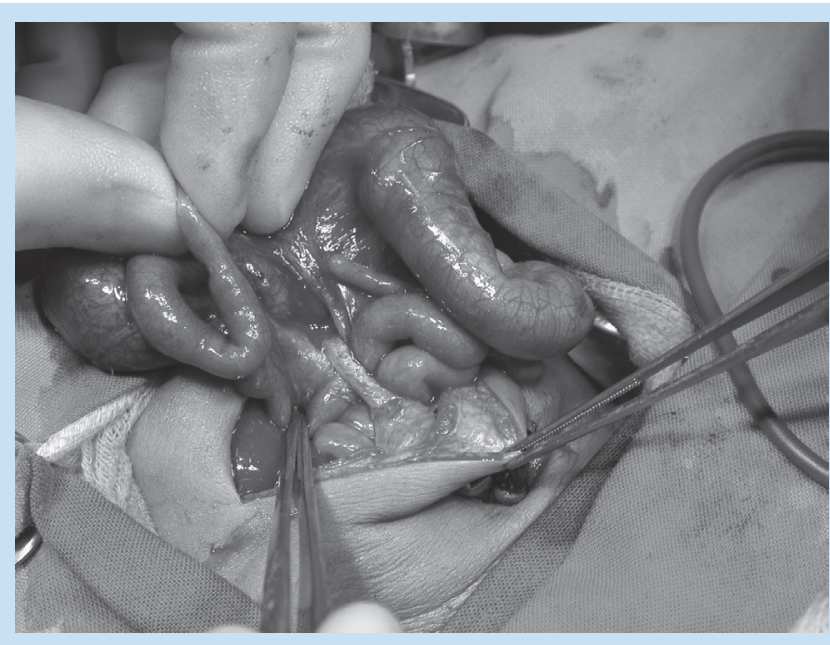

Fig. 2. At surgery the umbilical vein is seen draining into the superior mesenteric vein.

the region of the developing liver. They merge with the vitelline veins to form sinusoids.

The right umbilical vein regresses along with the part of the left umbilical vein that is nearest to the heart. The distal part of the left umbilical vein is left to become the main channel through the liver. It forms an anastamosis with the right hepatocardiac channel. By 3 months the right hepatocardiac channel enlarges and is termed the ductus

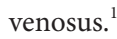

The ductus venosus connects the portal and umbilical veins with the inferior vena cava and acts as a sphincter to protect the fetus from placental overcirculation. The ductus venosus regresses at birth to form a fibrous cord, the ligamentum venosum. Its absence may cause hydrops fetalis, and is associated with a high mortality rate, chromosomal anomalies and congenital malformations. ${ }^{2}$

In our patient there were a host of additional abnormalities. On the abdominal film (Fig. 1) the nasogastric tube is curled in the proximal oesophageal pouch; this was confirmed to be the common type of oesophageal atresia with a fistula. In addition vertebral abnormalities of the upper dorsal spine are present.

The poor abdominal gas pattern was in keeping with the clinical and surgical findings of an anorectal malformation, colonic atresia, malrotation and the incidental finding of a Meckel's diverticulum.

At surgery a horseshoe kidney was observed.

The infant also had left radial hypoplasia. The heart however was structurally normal.

In view of the high incidence of congenital defects associated with umbilical vein variations and particularly an aberrant right umbilical vein, when these are detected it is suggested that the patient should be carefully investigated for other congenital abnormalities.

1. Moore KL. The Developing Human. 7th ed. Philadelphia: WB Saunders, 2002: 262-280, 364-371.

2. Perles Z, Nir A, Nadjari M, et al. Absent ductus venosus in the fetus: Review of the literature and first report of direct umbilical venous drainage to the coronary sinus. Fetal Diagn Ther 2003; 18:247-251. 